\title{
First International South Caucasian Conference on Rare Diseases and Orphan Drugs
}

\author{
Scientific Meeting - October 7-8, 2010, Yerevan, Armenia
}

(C) Springer-Verlag 2010
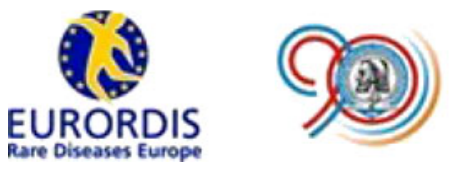

Dear Colleagues and Friends,

We have the pleasure to inform you that the First International South Caucasian Conference on Rare Diseases and Orphan Drugs will take place on October 7-8, 2010, at the Yerevan State Medical University (YSMU), Yerevan, Armenia.

The conference will be held within the frameworks of YSMU 90th Anniversary Congress. More information is available at (http://www.ysmu.am/90 and http://www.nhdmda.am/ISCORD\%202010).

Nowadays, problems concerning rare diseases are of a great importance not only to the countries of the European Community, the US, and many others countries of the World, but also to Armenia and the whole South Caucasian and Transcaucasian region. Therefore the conference is planned to be a regional one with participation of researchers, patients' representatives, policy makers, healthcare professionals, and industry representatives from Armenia and abroad.

Many participants from all over the world, more than 13 speakers and leaders in the field of rare diseases from US, Austria, France, Italy, Netherlands, and Switzerland, are expected to give main lectures. A number of International and European Health Organizations, such as the European Organization for Rare Diseases, the International PraderWilli Syndrome Organization (USA), have expressed their willingness to support the Conference.
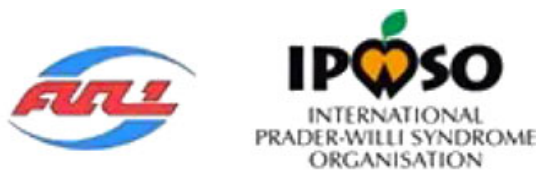

This is a good opportunity to bring together the energy, efforts, and knowledge of our scientists and other enthusiasts for this important initiative. This is a crucial moment for mobilizing our efforts to draw the attention of public health organizers, health-care professionals, and other authorities to approach the problem of rare diseases.

The conference will focus on the ways and strategies of cooperation to solve the problem of rare diseases in the Republic of Armenia and the whole South Caucasian region.

We hope that participants will enjoy not only the collaboration and exchange of experience, knowledge, and discussions, but that they also have the possibility to visit our old country with its modern as well as historical monuments of culture.

Your stay will be during a beautiful season with good and warm weather, too.

We are convinced that you will enjoy the visit of our republic, that you will receive many good and helpful information and impressions, and find a lot of good friends.

Welcome to Armenia!

Chairman of the Conference

Prof. Albert Matevosyan MD, PhD 